\title{
Plasticity of parahydrogen with reduced deuterium contents
}

\author{
L.A. Alekseeva \\ B. Verkin Institute for Low Temperature Physics and Engineering National Academy \\ of Sciences of Ukraine, 47 Lenin Ave., Kharkov 61103, Ukraine \\ E-mail: alekseeva@ilt.kharkov.ua \\ Received September, 2006
}

\begin{abstract}
The plasticity of solid parahydrogen with lowered deuterium contents under step-wise uniaxial extension at liquid helium temperatures $1.8-4.2 \mathrm{~K}$ has been investigated. Work hardening curves for single crystals have been measured. Maximum possible values of sample's elongation without their fracture at minimum stress values have been reached. Features of super-plastic irreversible deformation of samples were observed. Anomalous temperature dependence of deformation parameters has been found. The character of this anomaly exhibits evidence of the coherent motion of dislocation kinks in Pierls relief, modified by residual ortho- and deuterium impurities.
\end{abstract}

PACS: 62.20.-x Mechanical Properties of Solids; 67.80.-s Solid Helium and Related Quantum Crystals.

Keywords: hardening curve, yield-stress, coherent band, dislocation kink, fracture.

\section{Introduction}

Due to delocalization of the wave function, the particles that form a quantum (including parahydrogen) crystal are able to penetrate by tunneling through potential barriers, separating one equilibrium position in the lattice from another [1]. A possible crossover of a quantum crystal to a state with zero static shear modulus [2] can be observed by measuring its plasticity.

Pure $p-\mathrm{H}_{2}$ is a very plastic material down to $1.8 \mathrm{~K}$, i.e., about $1 \%$ of the Debye temperature [3]. It's deformation is brought about by extremely small loads [4-6]* and can be interpreted more adequately in epy quasi-particle approach. Isotopic impurities affect considerably the quantum nature of hydrogen crystal flow. Unlike the soft pure material, $p-\mathrm{H}_{2}$ doped with its stable deuterium isotope exhibit a considerable strengthening and a much lower plasticity $[4,5,8]$. Some other intriguing findings [6,9] on very pure $p-\mathrm{H}_{2}$ were tentatively interpreted as being related with large-scale planar defects**, viz., pseudotwins $[9,10]$. In view of this strong influence of isotopes, it would be nice to have a method enabling the purification of $p-\mathrm{H}_{2}$ in situ in order to obtain more perfect samples. In this work a new method is suggested for preparing single crystals of $p-\mathrm{H}_{2}$ with reduced isotope contents.

\section{Experimental}

Pure $p-\mathrm{H}_{2}$ was produced from normal hydrogen $\left(n-\mathrm{H}_{2}\right.$, $75 \% o-\mathrm{H}_{2}$ ), purified in a SHPV-500 reactor, according to the reactor certificate, to a level with less than $1 \mathrm{ppm}$ of non-hydrogen impurities. The $o-\mathrm{H}_{2}$ fraction was further reduced to $\sim 0.2 \%$ by keeping $n-\mathrm{H}_{2}$ long enough in the presence of $\mathrm{Fe}(\mathrm{OH})_{3}$ at the $\mathrm{H}_{2}$ boiling temperature.

Part of $p-\mathrm{H}_{2}$ was crystallized in a modified helium-cooled cell of the cryostat [11]. The crystallization completed, electric power was fed to reheat the cell again to $T \geq 15 \mathrm{~K}$. After the sample melted, part of the $p-\mathrm{H}_{2}$ vapor was redirected back to the converter. As a result of the difference between the partial pressures of deuterium and hydrogen, the contents of the less volatile component is

* Our earlier results on the gravity-related low-temperature flow of the crystal [5] are at variance with subsequent experiments [6,7].

** Occurrence of these planar defects in HCP $p-\mathrm{H}_{2}$ is caused by the small energy difference between HCP and FCC structures. 
naturally reduced, thereby enriching the more volatile component. Single-crystalline samples were grown from pure vapor above the $p-\mathrm{H}_{2}$ distillate after double rectification at a rate of $\sim 0.3-0.5 \mathrm{~mm} / \mathrm{min}$; the final heavier isotope fraction was considerably reduced (to $[\mathrm{D}] /[\mathrm{H}]$ $\sim 0.005-0.006 \%)$ compared to its natural abundance ([D]/[H] 0.0147-0.0156\% [12]). The considerable reduction of the isotope contents has been confirmed to within $30 \mathrm{ppm}$ by numerous isotope analyses by a modified MX-7304 mass spectrometer.

Transparent single-crystalline samples thus grown were separated from cell walls by pumping off the vapor over them, then annealed near the melting-point for $40-50 \mathrm{~min}$ and cooled slowly down to the test temperature.

Loading of samples was done with the aid of a $200 \mathrm{mG}$ sensitive balance scales. Crystal elongations were measured by the inductance displacement sensor with an accuracy of $\pm 1 \cdot 10^{-5} \mathrm{~cm}$, the temperature was measured with semiconductor thermometers to within $20 \mathrm{mK}$.

\section{Results and discussion}

Since planar defects can be easily identified [13], samples were first load-relieve cycle tested. Figure 1 presents a typical dependence of the relative elongation $\varepsilon$ on the applied stress $\sigma$. This stress-strain curve $\sigma(\varepsilon)$ was obtained by a stepwise stretching. A fully irreversible character of deformation (no hysteresis observed) implied absence of large-scale planar defects. Reverse movement of the rod resulted in loop-like macroscopic deformations resembling observed earlier [5]

Figure 2 presents initial stages of the $\sigma(\varepsilon)$ curves, which correspond to the boundary temperatures studied $(T=4.2$ and $1.8 \mathrm{~K})$. The strain $\varepsilon$ here is limited along the horizontal axis by the lowermost value observed ( $\sim 50 \%)$ in experiment. Every crystal was tested (without fracture) only at one temperature. Depending on the temperature value, 7 to 9 crystals were examined. The two $\sigma(\varepsilon)$ curves

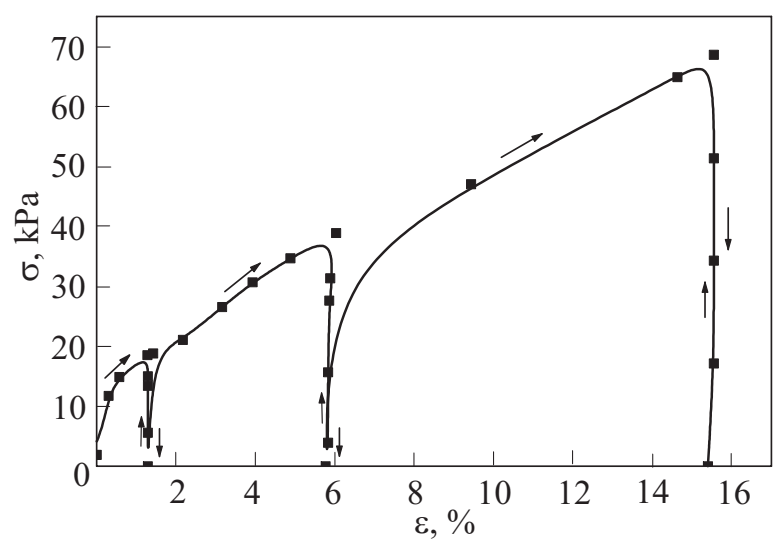

Fig. 1. Typical $\sigma(\varepsilon)$ obtained during load-relieve cycling for $p-\mathrm{H}_{2}([\mathrm{D}] /[\mathrm{H}] \sim 50-60 \mathrm{ppm})$ at $T=4.2 \mathrm{~K}$.

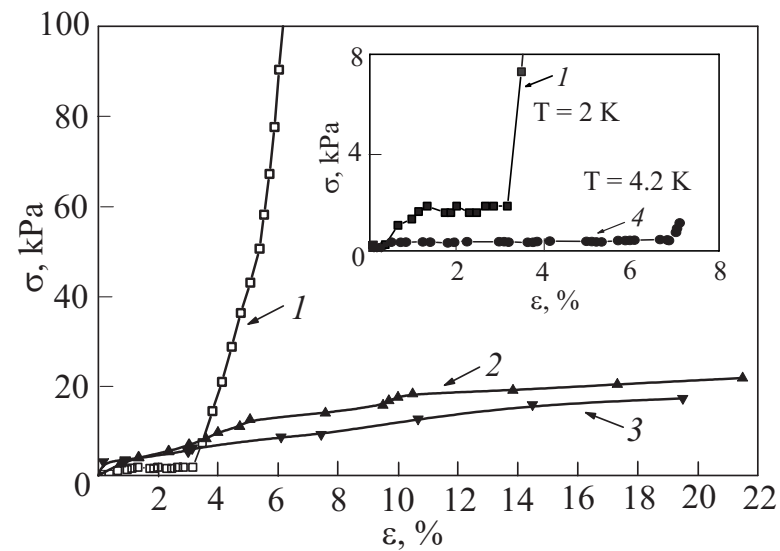

Fig. 2. Typical $\sigma(\varepsilon)$ curves, measured on single crystals of parahydrogen: curves 2 and 3 are data of this work $\left(o-\mathrm{H}_{2} \sim\right.$ $\sim 0.2 \%,[\mathrm{D}] /[\mathrm{H}] \sim 50-60 \mathrm{ppm}$, nonhydrogen impurities $1 \mathrm{ppm} ;$ $T=4.2 \mathrm{~K}(2), T=1.8 \mathrm{~K}(3)$; curves 1 and 4 are compression data [6] for $2 \mathrm{~K}$ and $4.2 \mathrm{~K}$ for super-pure $p-\mathrm{H}_{2}\left(o-\mathrm{H}_{2} \sim 0.01-0.005 \%\right.$, $[\mathrm{D}] /[\mathrm{H}] \sim 1 / 5-1 / 7$ of the natural abundance, nonhydrogen impurities $\sim 0.002 \%$ ).

in Fig. 1 plotted from data obtained on those samples which showed maximum strain at minimum stress, which correspond to the most favourable orientation of the basal HCP plane relatively to the stretching axis. The second longer (almost linear) stages (not shown in Fig. 2) are characterized by small of strengthening coefficients $\theta=d \sigma / d \varepsilon$ and by minimum stresses $\sigma_{0}$ of the stage to stage crossover. The $\theta$ values, normalized to the shear modulus of solid $p-\mathrm{H}_{2}[3]$ were $3 \cdot 10^{-5}$ and $2.5 \cdot 10^{-5}$ at 4.2 $\mathrm{K}$ and $1.8 \mathrm{~K}$, respectively. Such low values of $\theta$ are typical of the developed flow stage of the materials with HCP structure [14], including $p-\mathrm{H}_{2}$ single crystals [5]. They demonstrate that the $p-\mathrm{H}_{2}$ deformation occurs due to dislocation displacements in any one of the possible crystallographic planes [14]. It is interesting that the $\sigma(\varepsilon)$ curve in Fig. 2 for the lower $T$ is below the curve for the higher $T$, which contradicts the standard notion of thermally activated deformation processes. The same concerns the $\sigma_{0}$ versus $T$ relation. The anomalous character of the yield stress is evidence that quantum tunneling mechanisms definitely prevails over classical thermally activated processes. For comparison, $\sigma(\varepsilon)$ curves measured under compression of single crystals of $p-\mathrm{H}_{2}$ at $2 \mathrm{~K}$ and $4.2 \mathrm{~K}$ [6] are also shown in Fig. 2. Note that quite small stresses are needed to obtain of comparatively great $(\sim 3-7 \%)$ strains. But further compression leads to a strong strengthening of samples. It is possible that the twins (or other packing faults), which are presumably responsible for the hysteresis first observed in Ref. 6, lead to the strong hardening in their samples. It should be remarked that their samples were extremely pure if ortho-and isotope impurities are concerned but contained much more $(20 \mathrm{ppm})$ non-hydrogen impurities, which are capable of influencing crucially the plasticity of solid hydrogen. 
Thus, the data presented here suggest that the plastic flow is provided by individual dislocations moving through the lattice via some tunneling mechanism, like it was claimed in Ref. 5. Our present results give also evidence that an important role in plastic flow belongs to microscopic defects (vacancies and dislocation kinks) and their coherent band motion.

Since our samples contained low ortho-fractions (about $0.2 \%$ ), the number of pairwise ortho-clusters that can interact significantly with moving dislocations (see [15]) was small number (of the order of $2 \cdot 10^{-6}$ ). Therefore, the main obstacle for dislocation motion was the Pierls relief, modified by the deuterium impurities, which might be efficient stoppers.

Summing up, the main agent that provides the dislocation motion in the $p-\mathrm{H}_{2}$ crystals studied is, most likely, dislocation kink $[16,17]$. The anomalous character of the temperature dependency of both the yield stress $\sigma_{0}(T)$ and the hardening coefficient is evidence of a band-like character of their motion.

The author thanks M.A. Strzhemechny, K.A. Chishko, A.I. Prokhvatilov, and V.D. Natsik for the discussions of our results, D.N. Kazakov (Moscow, Russia) for numerous mass-spectrometric and chromatographic analyses, L.A. Vashchenko and T.Ph. Lemzyakova for chromatographic analysis, and A.V. Kuznetsov for helping with experiments.

1. A.F. Andreev and I.M. Lifshits, Zh. Eksp. Teor. Fiz. 56, 2057 (1969) [Sov. Phys. JETP 29, 1107 (1969)]; A.F. Andreev, Usp. Fiz. Nauk 118, 251 (1976) [Sov. Phys. Usp. 19, 137 (1976)].

2. I.M. Lifshits, Fiz. Nizk. Temp. 1, 896 (1975) [Sov. J. Low Temp. Phys. 1, 429 (1975)].
3. Physics of Cryocrystals, V.G. Manzhelii, Yu.A. Freiman, M.L. Klein, and A.A. Maradudin (eds.), AIP Press, Woodbury, New York (1996).

4. I.N. Krupskii, A.V. Leont'eva, L.A. Indan, and O.V. Evdokimova, Pis'ma Zh. Eksp. Teor. Phys. 24, 297 (1976) [Sov. JETP Lett. 24, 266 (1976)].

5. L.A. Alekseeva and I.N. Krupskii, Fiz. Nizk. Temp. 10, 327 (1984) [Sov. J. Low Temp. Phys. 10, 170 (1984)].

6. A.N. Aleksandrovskii, E.A. Kir'yanova, V.G. Manzhelii, A.V. Soldatov, and A.M. Tolkachev, Fiz. Nizk. Temp. 13, 1095 (1987) [Sov. J. Low Temp. Phys. 13, 623 (1987)].

7. Q. Xiong and H.J. Maris, J. Low Temp. Phys. 81, 167 (1990).

8. L.A. Alekseeva, E.S. Syrkin, and L.A. Vashchenko, Fiz. Tverd. Tela (St. Peterburg) 45, 1024 (2003) [Sov. Phys. Solid State 45, 1073 (2003)].

9. L.A. Alekseeva, V.D. Natsik, B.A. Halin, and A.V. Pustovalova, Fiz. Nizk. Temp. 14, 1127 (1988) [Sov. J. Low Temp. Phys. 14, 621 (1988)].

10. K.A. Chishko, Fiz. Nizk. Temp. 15, 106 (1989) [Sov. J. Low Temp. Phys. 15, 61 (1989)].

11. I.N. Krupskii, A.V. Leont'eva, L.A. Indan, and O.V. Evdokimova, Fiz. Nizk. Temp. 3, 933 (1977) [Sov. J. Low Temp. Phys. 3, 453 (1977)].

12. V.M Andreev, Yu.A. Zel'vinskii, and S.G. Katal'nikov, Heavy Hydrogen Isotopes in Nuclear Engeneering, Energoatomizdat, Moscow (1987) [in Russian].

13. V.S. Boiko, R.I. Garber, and A.M. Kosevich, Reversible Plasticity of Crystalls, Nauka, Moscow (1991) [in Russian].

14. J. Friedel, Dislocations, Pergamon, Oxford (1964); Mir, Moscow (1967).

15. S.E. Kal'noi and M.A. Strzhemechny, Fiz. Nizk. Temp. 11, 803 (1985) [Sov. J. Low Temp. Phys. 11, 440 (1985)].

16. B.V. Petuhov and V.L. Pokrovskii, Pis'ma Zh. Eksp. Teor. Phys. 15, 63 (1972) [Sov. JETP Lett. 15, 44 (1972)].

17. M.A. Strzhemechny, Fiz. Nizk. Temp. 10, 663 (1984) [Sov. J. Low Temp. Phys. 10, 348 (1984)]. 